\title{
CONTENTS OF THE DVD
}

The main reference to each DVD clip appears in boldface in the text.

Clip 1.1. Signed Introduction, by Ben Bahan

Clip 1.2. "Let There Be Light," by H-Dirksen L. Bauman, signed by

Danny Lucero

Clip 1.3. Signs for MUSIC and POETRY

Clip 1.4. Signs for EXPRESSION and SIGN-POETRY

Clip 1.5. Sign for ORAL-TRADITION

Clip 1.6. “'hydrogen jukebox,' translated by Patrick Graybill,” signed by Peter Cook

Clip 2.1. "BOAT, BOAT, BOAT-BOAT-BOAT," signed by George Kannapell

Clip 2.2. "Ball Story," by Ben Bahan

Clip 2.3. "Durassic Park," by Manny Hernandez

Clip 2.4. "Eyeballs Popping Out . . .," signed by Ben Bahan

Clip 2.5. For a Decent Living, opening segment, by Sam Supalla

Clip 2.6. "Civil War Story," signed by Ben Bahan

Clip 2.7. "Star Spangled Banner," signed by Mrs. Washington Barrow

Clip 2.8. "Star Spangled Banner," signed by Ella Mae Lentz

Clip 2.9. "Virginia School for the Deaf's Fight Song," signed by Freda Norman

Clip 2.10. "Haunted House" ABC story, by Ben Bahan

Clip 2.11. "Haunted House," ABC segment, signed by Ben Bahan 
Clip 2.12. "Race," ABC segment, by Ben Bahan

Clip 2.13. "A Full Hand," by Freda Norman

Clip 5.1. "ASL Rhymes, with an excerpt from Clayton Valli's

'Snowflake," signed by E. Lynn Jacobowitz

Clip 5.2. "Snowflake," by Clayton Valli

Clip 5.3. "Eye Music," by Ella Mae Lentz

Clip 5.4. "Poetry," by Flying Words Project

Clip 5.5. "Visual Vernacular," signed by Peter Cook

Clip 5.6. "Cinematic Techniques in ASL," by Manny Hernandez

Clip 5.7. "Times Squared," by Manny Hernandez

Clip 7.1. "Travels with Malz: The Next Generation," by Ella Mae Lentz

Clip 7.2. "Silence Oh Painful," by Ella Mae Lentz

Clip 7.3. "Missing Children," by Debbie Rennie, signed by Debbie Rennie

Clip 7.4. "Missing Children," by Debbie Rennie, signed by Missy Keast

Clip 7.5. "Dew on Spiderweb," by Clayton Valli, signed by Ella Mae Lentz

Clip 8.1. "Process Overview," by Peter Cook

Clip 8.2. "Old Time Airplane," by Peter Cook

Clip 8.3. "Transformation Technique," by Peter Cook

Clip 8.4. "Samples of Student Poems," signed by Peter Cook

Clip 8.5. "Reflections on the Signing Process," by Peter Cook

Clip 9.1. "Epic: Gallaudet Protest," by Gilbert C. Eastman

Clip 10.1. "Loudest Visual Scream," by Willy Conley

Clip 10.2. "Sign-Mime," by Willy Conley

Clip 10.3. "Charlie Chaplin and City Lights," by Willy Conley

Clip 11.1. "I Am Ordered Now to Talk," by Flying Words Project

Clip 11.2. "Wave," by Aaron Williamson

Clip 11.3. Hearing Things, by Aaron Williamson 